\title{
Effects of smoking cessation on central blood pressure and arterial stiffness
}

This article was published in the following Dove Press journal:

Vascular Health and Risk Management

19 October 2011

Number of times this article has been viewed

\author{
Takeshi Takami' \\ Yoshihiko Saito ${ }^{2}$ \\ 'Department of Internal Medicine, \\ Clinic Jingumae, Kashihara, Nara, \\ Japan; ${ }^{2}$ First Department of Internal \\ Medicine, Nara Medical University, \\ Kashihara, Nara, Japan
}

Correspondence: Takeshi Takami Department of Internal Medicine, Clinic Jingumae, 5-4-4I Naizencho, Kashihara, Nara 634-0804, Japan

Tel +8 I 744238568

Fax +8I 744236818

Email takami66@m5.kcn.ne.jp
Purpose: Smoking affects arterial stiffness, thus causing an elevation in central blood pressure (CBP). The present study was designed to examine whether smoking cessation treatment improved CBP and arterial stiffness.

Patients and methods: We conducted an observational study of 70 patients receiving smoking cessation treatment. Before and 60 weeks after the start of a 12-week varenicline treatment, we measured brachial blood pressure, CBP, brachial-ankle pulse wave velocity (baPWV), normalized radial augmentation index (rAIx@75), left ventricular weight, and left ventricular diastolic function of each patient. The data were compared between the patients who succeeded in quitting smoking (smoking cessation group; $\mathrm{n}=37$ ) and those who failed to quit smoking (smoking group; $\mathrm{n}=33$ ).

Results: Baseline characteristics were similar in both groups. Brachial blood pressure remained unchanged in both groups. CBP, baPWV, and rAIx@75 decreased significantly in the smoking cessation group, while these parameters showed no significant change in the smoking group. Thus, CBP, baPWV, and rAIx@75 showed greater decrease in the smoking cessation group than in the smoking group (CBP, $-7.1 \pm 1.4 \mathrm{mmHg}$ vs $1.2 \pm 2.7 \mathrm{mmHg} ; P<0.01 ; \mathrm{baPWV},-204 \pm 64 \mathrm{~cm} / \mathrm{s}$ vs $-43 \pm 72 \mathrm{~cm} / \mathrm{s} ; P<0.01 ;$ rAIx@75, $-6.4 \pm 2.8 \%$ vs $-1.0 \pm 3.9 \% ; P<0.01)$. Left ventricular weight and left ventricular diastolic function remained unchanged in both groups.

Conclusion: Patients in the smoking cessation group showed significant improvement in CBP, baPWV, and rAIx@75. These results indicate that smoking cessation can improve arterial stiffness and CBP.

Keywords: central blood pressure, augmentation index, brachial-ankle pulse wave velocity, smoking cessation, varenicline

\section{Introduction}

Smoking induces a temporary elevation in blood pressure. The elevation in blood pressure induced by smoking one cigarette lasts for a period of 15 minutes or more. ${ }^{1}$ Blood pressure may remain elevated in heavy smokers. Habitual smokers show elevation in blood pressure during daytime activities, ${ }^{2}$ or they show an elevation in central blood pressure (CBP). ${ }^{3} \mathrm{CBP}$ induces a more direct mechanical stress on the left ventricle, large arteries, and vital organ vasculature than does brachial blood pressure. The impact of CBP has been reported in large-scale intervention trials and population-based studies, such as the CAFÉ-ASCOT study ${ }^{4}$ and the SHS study. ${ }^{5}$ In addition, smoking might cause masked hypertension. ${ }^{6}$ However, a detailed evaluation of the influence of chronic smoking on blood pressure has not been performed thus far. ${ }^{7}$ The relationship between smoking cessation and arterial wall thickness and 
stiffness has been reported by van den Berkmortel et al. ${ }^{8} \mathrm{CBP}$ can now be measured noninvasively, and CBP measurement using such methods has yielded many new findings. It is well known that elevation in CBP, rather than elevation in brachial blood pressure, is more closely associated with the onset of cardiovascular events. ${ }^{9}$ The impact of smoking cessation with nicotine replacement on arterial stiffness remains unknown. In recent years, drug therapy with varenicline, an $\alpha 4 \beta 2$ nicotinic acetylcholine receptor partial agonist, has been actively used in helping smokers to quit smoking. ${ }^{10,11}$ The present study was undertaken to evaluate the influence of smoking cessation with varenicline on CBP.

\section{Methods}

\section{Patients and study design}

We conducted an observational study for 1 year on 70 patients receiving smoking cessation treatment at our clinic. Brachial blood pressure, CBP, radial augmentation index (AI), brachial-ankle pulse wave velocity (baPWV), left ventricular weight, and left ventricular diastolic function were measured before and 60 weeks after the start of the smoking cessation treatment. Varenicline, which was administered for 12 weeks ( $0.5 \mathrm{mg}$ once daily for 3 days, $0.5 \mathrm{mg}$ twice daily for 4 days, and then $1.0 \mathrm{mg}$ twice daily for a total of 12 weeks), was used for smoking cessation. The carbon monoxide (CO) level in expired gas was measured at 2-week intervals using a piCO Smokerlyzer (Bedfont, Kent, UK) to check whether the patients had quit smoking. Of the 70 patients, 56 completed the 12 -week treatment. Of these 56 patients, 19 resumed smoking during the treatment period. During the 1-year observational study after the end of smoking cessation, data were analyzed by dividing the patients into a smoking cessation group (37 patients who quit smoking until 1 year after the end of treatment) and a smoking group (33 patients who resumed smoking).

All patients were informed about the study procedure. Written informed consent was obtained from all patients participating in the study. The study protocol was approved by the Institutional Ethics Committee.

Brachial blood pressure, CBP, and radial AI were measured by radial tonometry by using HEM9000-AI (Omron Healthcare, Kyoto, Japan). Because the radial AI is affected by meals, it was measured on an empty stomach. To account for the influence of heart rate (HR), radial AI was corrected for $\mathrm{HR}(75 / \mathrm{m})$ and expressed as rAIx@75. The baPWV was determined from the pulse waveforms recorded from both forearms and both ankles using the formula PWV (Omron Healthcare, Kyoto, Japan). Left ventricular weight and left ventricular diastolic function were measured by echocardiography (VIVID ${ }^{\text {TM }}$ S6; GE Healthcare, Milwaukee, WI). Left ventricular weight was determined from the M-mode ultrasound image by using the equation of Devereux. ${ }^{12}$ Left ventricular mass index (LVMI) was calculated by dividing the left ventricular weight by body surface area of the patient. Left ventricular diastolic function was evaluated by measuring mitral annulus velocity $\left(\mathrm{e}^{\prime}\right)$ at early diastole by tissue Doppler imaging.

\section{Measurement of CBP and rAlx@75}

The pulse pressure waveform of the radial artery was recorded using an automated tonometry system (HEM9000AI; Omron Healthcare, Kyoto, Japan) with the patient in a sitting position after resting for $\geq 5$ minutes. The waveform was automatically calibrated using the built-in oscillometric brachial sphygmomanometer, and the peak and trough of the radial pressure wave were adjusted to brachial systolic blood pressure (SBP) and diastolic blood pressure (DBP), respectively. The second peak (late systolic inflection) was automatically detected by an algorithm programmed into the HEM-9000AI system using the second maxima of the fourth derivative of the radial pressure waveform to determine the radial AI as well as the late or second SBP (SBP2). This algorithm is described in more detail elsewhere. ${ }^{13}$ The height of the second peak corresponds to the SBP2 value obtained using HEM-9000AI. The value of SBP2 is very similar to that of aortic CBP recorded using invasive techniques, ${ }^{14}$ and thus, SBP2 was used as an estimate of CBP. CBP determined using HEM9000-AI was comparable to that determined using a generalized aorta-radial transfer function. ${ }^{15,16}$ The AI was calculated using the following formula: (SBP2 - DBP)/ (the first peak SBP - DBP) $\times 100$. Because AI is influenced by the HR, AI was normalized for a HR of 75 bpm (rAIx@75), as proposed by Wilkinson et al. ${ }^{17}$ In this study, these measurements were performed by a single expert investigator.

\section{Measurement of baPWV}

The baPWV was also used to assess arterial stiffness. Measurement of baPWV was performed as described previously. ${ }^{18}$ Briefly, baPWV was determined from the pulse waveforms recorded from both forearms and both ankles using the formula PWV (Omron Healthcare, Kyoto, Japan). The baPWV was measured in patients who had been lying in a supine position for at least 5 minutes. Measurements of baPWV were repeated twice to confirm reproducibility, and the deviation between measurements was within 5\%. The mean value on the right side was used as the baPWV value in each patient. 
In this study, this measurement was performed by a single expert investigator.

\section{Measurement of LVMI and e'}

M-mode echocardiography was performed under twodimensional echocardiography guidance using a Vivid S6 ultrasound system (GE Healthcare, Milwaukee, WI) with a 3-MHz transducer. LVMI was measured by standard M-mode echocardiography and determined using the formula reported by Devereux et al. ${ }^{12}$ Longitudinal velocity of the mitral annulus was recorded throughout the cardiac cycle at the septal annulus in the apical four-chamber view by spectral pulsed-wave Doppler imaging.

The peaks of systolic apically directed ( $\mathrm{s}^{\prime}$ velocity) and early diastolic ( $\mathrm{e}^{\prime}$ velocity) myocardial velocities were measured.

\section{Statistical analysis}

Data are shown as means \pm SD. Differences between the two groups at baseline were analyzed using unpaired $t$-tests for continuous variables and $\chi^{2}$ tests for categorical variables. Paired $t$-tests were used for within-group comparisons, and unpaired $t$-tests were used for between-group comparisons. Values of $P<0.05$ (two-sided) were considered statistically significant. SAS software (v8.2; SAS Institute Inc, Cary, NC) was used for all analyses.

\section{Results}

No significant difference was observed between the two groups in any background variable, including background variables known to affect $\mathrm{CBP}$ and $\mathrm{AI}$, such as age, renal function, diabetes mellitus, dyslipidemia, gender, and BMI (Table 1). About $30 \%$ of the patients had hypertension. No hypotensor was used in patients with systolic blood pressure below $150 \mathrm{mmHg}$.

In the smoking cessation group, the $\mathrm{CO}$ level in expired gas decreased from $3.8 \pm 0.4 \%$ to $0.4 \pm 0.1 \%(P<0.001)$, while that in the smoking group did not change $(3.6 \pm 0.5 \%$ before smoking cessation and $3.7 \pm 0.5 \%$ after smoking cessation, $P=0.65)$. The change in the $\mathrm{CO}$ level in expired gas following treatment differed significantly between the two groups $(P<0.001)$.

SBP measured at the brachium showed no significant reduction after smoking cessation in either of the two groups, and the brachial SBP was not significantly different between the two groups. DBP measurement showed results similar to those of SBP. In smoking cessation group, the SBP was $138.3 \pm 6.4 \mathrm{mmHg}$ before smoking cessation and $136.7 \pm 6.4 \mathrm{mmHg}$ after smoking cessation. In the smoking
Table I Baseline characteristics

\begin{tabular}{|c|c|c|c|}
\hline & $\begin{array}{l}\text { Smoking } \\
\text { cessation group } \\
(n=37)\end{array}$ & $\begin{array}{l}\text { Smoking } \\
\text { group } \\
(n=33)\end{array}$ & $P$ value \\
\hline Age (years) & $51.3 \pm 5.9$ & $53.5 \pm 7.9$ & 0.48 \\
\hline Sex (male, \%) & 69 & 71 & 0.72 \\
\hline BMI $\left(\mathrm{kg} / \mathrm{m}^{2}\right)$ & $24.5 \pm 2.1$ & $23.9 \pm 2.5$ & 0.54 \\
\hline $\mathrm{SBP}(\mathrm{mmHg})$ & $138.3 \pm 6.4$ & $135.9 \pm 7.3$ & 0.42 \\
\hline $\mathrm{DBP}(\mathrm{mmHg})$ & $85.4 \pm 3.1$ & $84.2 \pm 4.6$ & 0.64 \\
\hline $\mathrm{CBP}(\mathrm{mmHg})$ & $109.4 \pm 2.1$ & $110.1 \pm 2.9$ & $0.7 I$ \\
\hline HR (bpm) & $71.3 \pm 5.3$ & $68.4 \pm 6.3$ & 0.54 \\
\hline $\mathrm{baPWV}(\mathrm{cm} / \mathrm{s})$ & $1768.3 \pm 54.5$ & $1802.5 \pm 73.8$ & 0.37 \\
\hline rAlx@75 (\%) & $77.5 \pm 3.4$ & $77.9 \pm 3.8$ & 0.21 \\
\hline LVMI $\left(g / m^{2}\right)$ & $113.6 \pm 5.5$ & $\mid 16.1 \pm 3.6$ & 0.16 \\
\hline $\begin{array}{l}\text { eGFR } \\
\left(\mathrm{ml} \cdot \mathrm{min}^{-1} \cdot 1.73 \mathrm{~m}^{-2}\right)\end{array}$ & $65.3 \pm 3.5$ & $67.1 \pm 4.8$ & 0.21 \\
\hline $\mathrm{HbA}_{\mathrm{lc}}(\%)$ & $5.1 \pm 0.3$ & $5.0 \pm 0.4$ & 0.51 \\
\hline LDL-C (mg/dL) & $105.6 \pm 6.3$ & $106.9 \pm 8.9$ & 0.55 \\
\hline HDL-C (mg/dL) & $48.6 \pm 4.8$ & $51.2 \pm 5.8$ & 0.16 \\
\hline TG (mg/dL) & $105.8 \pm 15.7$ & $110.8 \pm 13.8$ & 0.34 \\
\hline UA (mg/dL) & $5.4 \pm 0.9$ & $5.6 \pm 0.6$ & 0.51 \\
\hline $\mathrm{e}^{\prime}(\mathrm{cm} / \mathrm{s})$ & $9.5 \pm 0.5$ & $9.7 \pm 0.7$ & 0.34 \\
\hline
\end{tabular}

Note: Values are means \pm standard deviation or $n(\%)$.

Abbreviations: BMI, body mass index; SBP, systolic blood pressure; DBP, diastolic blood pressure; $C B P$, central blood pressure; HR, heart rate; baPWV, brachialankle pulse wave velocity; rAlx@75, normalized augmentation index; LVMI, left ventricular mass index; eGFR, estimated glomerular filtration rate; LDL-C, low density lipoprotein cholesterol; HDL-C, high density lipoprotein cholesterol; TG, triglyceride; UA, uric acid; e', mitral annulus velocity.

group, the SBP was $135.9 \pm 7.3 \mathrm{mmHg}$ before treatment and $134.8 \pm 6.3 \mathrm{mmHg}$ after smoking cessation. No significant difference was observed in DBP and HR between the two groups.

CBP, which represents the blood pressure near the origin of the aorta, decreased significantly in the smoking cessation group from $109.4 \pm 2.1 \mathrm{mmHg}$ before smoking cessation to $102.3 \pm 1.7 \mathrm{mmHg}$ after smoking cessation. CBP remained unchanged $(110.1 \pm 2.9 \mathrm{mmHg}$ before smoking cessation and $111.3 \pm 2.8 \mathrm{mmHg}$ after smoking cessation) in the smoking group. Thus, the change in CBP after smoking cessation differed significantly between the two groups. Figure 1 shows the changes in $\mathrm{CBP}$ after smoking cessation from its baseline value. The figure shows significant reduction of $\mathrm{CBP}$ in the smoking cessation group than in the smoking group.

Values for rAIx@75 showed similar trends to those for CBP; rAIx@75 (an indicator of arterial stiffness) decreased significantly in the smoking cessation group after smoking cessation $(77.5 \pm 3.4 \%$ before smoking cessation and $71.1 \pm 2.4 \%$ after smoking cessation).rAIx@75 remained unchanged in the smoking group $(77.9 \pm 3.8 \%$ before smoking cessation and $76.9 \pm 4.1 \%$ after smoking cessation). The change in rAIx@75 following treatment differed significantly 


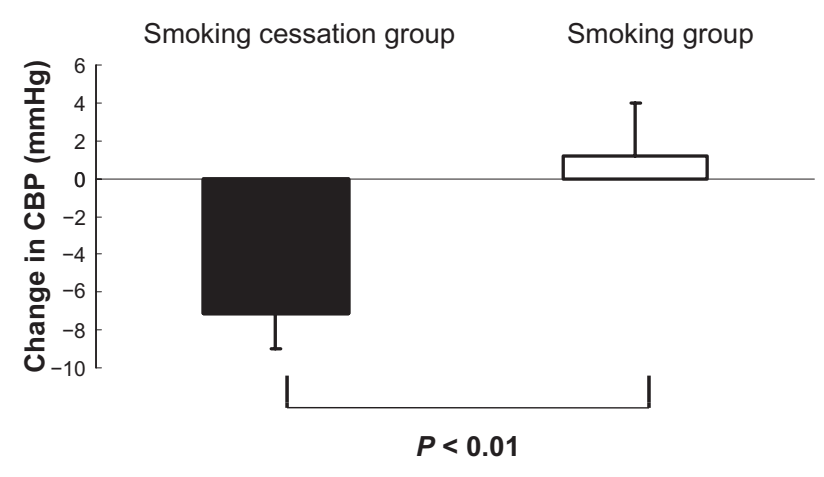

Figure I Changes in central blood pressure (CBP) from baseline to week 60

between the two groups. Figure 2 shows the changes in rAIx@75 after treatment from its baseline value.rAIx@75 decreased significantly after smoking cessation in the smoking cessation group than in the smoking group.

Results for baPWV were similar to those of rAIx@75; baPWV decreased significantly after smoking cessation in the smoking cessation group $(1768 \pm 54 \mathrm{~cm} / \mathrm{s}$ before smoking cessation and $1564 \pm 69 \mathrm{~cm} / \mathrm{s}$ after smoking cessation). baPWV remained unchanged $(1802 \pm 73 \mathrm{~cm} / \mathrm{s}$ before smoking cessation and $1759 \pm 85 \mathrm{~cm} / \mathrm{s}$ after smoking cessation) in the smoking group. The change in baPWV after treatment was significantly different between the two groups. Figure 3 shows the changes in baPWV after treatment from its baseline value. baPWV decreased significantly after smoking cessation in the smoking cessation group than in the smoking group.

LVMI, an indicator of left ventricular thickening, did not decrease significantly after smoking cessation in any of the two groups, and there was no significant difference in LVMI between the two groups. The LVMI in the smoking cessation group was $113.6 \pm 5.5 \mathrm{~g} / \mathrm{m}^{2}$ before treatment and $112.9 \pm 3.6 \mathrm{~g} / \mathrm{m}^{2}$ after smoking cessation and that in the smoking group was $116.1 \pm 3.6 \mathrm{~g} / \mathrm{m}^{2}$ before smoking cessation and $115.9 \pm 3.3 \mathrm{~g} / \mathrm{m}^{2}$ after smoking cessation.

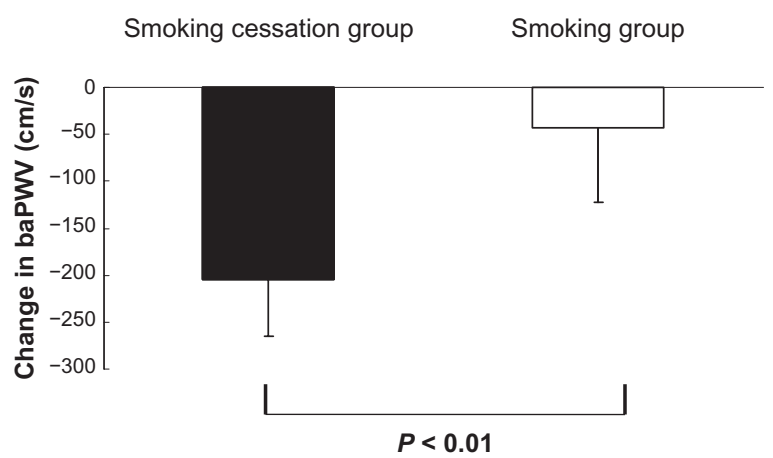

Figure 2 Changes in brachial-ankle pulse wave velocity (baPWV) from baseline to week 60 .

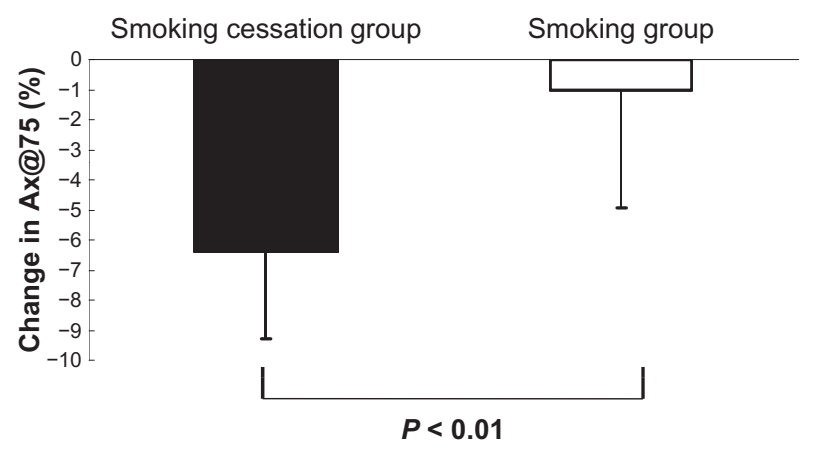

Figure 3 Changes in normalized augmentation index (rAlx@75) from baseline to week 60 .

The value of $\mathrm{e}^{\prime}$, an indicator of left ventricular diastolic function, did not decrease significantly after smoking cessation in any of the groups, and there was no significant difference in $\mathrm{e}^{\prime}$ between the two groups. In the smoking cessation group, $\mathrm{e}^{\prime}$ was $9.5 \pm 0.5 \mathrm{~cm} / \mathrm{s}$ before smoking cessation and $9.4 \pm 0.3 \mathrm{~cm} / \mathrm{s}$ after smoking cessation. In the smoking group, $\mathrm{e}^{\prime}$ was $9.7 \pm 0.7 \mathrm{~cm} / \mathrm{s}$ before smoking cessation and $9.6 \pm 0.6 \mathrm{~cm} / \mathrm{s}$ after smoking cessation.

\section{Adverse events}

All patients who entered the randomized phase of the study completed the study without experiencing any serious adverse events.

\section{Discussion}

Minami et al analyzed the relationship between smoking habits and AI, brachial blood pressure, and CBP in normal men, and reported that brachial blood pressure did not differ between smokers and nonsmokers, but CBP and AI were higher in smokers than in nonsmokers. ${ }^{3}$ Our results indicate that smoking cessation reduced CBP and AI. We did not include smokers with hypertension in our study; thus, it is necessary to examine whether smoking cessation reduces $\mathrm{CBP}$ and AI even in such patients. However, patients with hypertension are susceptible to the influence of drug therapy. Drugs such as renin angiotensin system inhibitors (angiotensin receptor blockers [ARB] and angiotensin-converting enzyme inhibitors [ACEI] and calcium channel blockers) are known to lower CBP and AI, while those such as diuretics and beta-blockers do not lower CBP or AI. ${ }^{4}$ To avoid such influences resulting from drug therapy, we included only those patients who did not receive hypotensors.

In the present study, smoking cessation did not affect left ventricular thickening or left ventricular diastolic function. This may be because reduction of afterload due to decrease in CBP and AI did not have a marked impact on left ventricular 
thickening or diastolic function in patients with normal blood pressure before smoking cessation.

The question arises of why did smoking cessation lower $\mathrm{CBP}$ and AI? Some investigators reported that AI is inversely correlated with flow-mediated vasodilatation, an indicator of endothelial function. ${ }^{19}$ Thus, the reduction in AI in our study may be because of an improvement in endothelial cell function. Mainstream cigarette smoke contains about $4 \%$ $\mathrm{CO}$, which binds firmly to blood hemoglobin. The $\mathrm{CO}$ bound to hemoglobin stimulates cholesterol degeneration, which results in impairment of the vascular endothelium and stimulation of atherosclerosis. ${ }^{20}$ The reduced $\mathrm{CO}$ level in expired gas observed after smoking cessation may be an indicator that this treatment decreases the level of hemoglobin-bound $\mathrm{CO}$. Tsuchiya et al showed that superoxides are the main reactive oxygen species contained in cigarette smoke. ${ }^{21}$ Smoking causes severe accumulation of exogenous active oxygen and free radicals, which results in chronic increase in oxidative stress, ${ }^{22}$ possibly leading to vascular endothelial dysfunction, vasoconstriction, and vascular inflammation. ${ }^{23}$ In our study, smoking cessation probably lowered the chronic oxidative stress. These changes might have improved the vascular endothelial dysfunction, leading to reduction in AI. Nicotine, contained in tobacco smoke, stimulates the adrenal gland, causing release of catecholamines and stimulation of the sympathetic nervous system and thus leading to contraction of peripheral vessels, elevation of blood pressure, and an increase in HR. ${ }^{24}$ In addition, nicotine stimulates the release of thromboxane $\mathrm{A} 2$, which has potent vasoconstrictive activity. ${ }^{25}$ These factors can lead to abnormal vasoconstriction. Smoking cessation had no effect on brachial blood pressure, indicating that this system was not involved in patients with normal blood pressure. Some investigators previously reported a study designed to evaluate the influence of acute and chronic smoking on arterial stiffness by analyzing baPWV and cardio-ankle vascular index (CAVI). ${ }^{26}$ Their results showed a significant elevation in baPWV after smoking.

Chronic smoking is thought to affect CBP through its effects on arterial stiffness and wave reflection. ${ }^{27}$ The results from our study suggest that improvement in baPWV and AI delayed the pulse-wave velocity and suppressed the increase in wave reflection, resulting in reduction of CBP.

Van den Berkmortel et $\mathrm{al}^{8}$ analyzed the relationship between smoking cessation and arterial wall thickness and stiffness and reported that even after 2 years of smoking cessation, the intima-media thickness did not reduce and no improvement was observed in the cross-sectional compliance or distensibility coefficient. In our study, we did not estimate the cross-sectional compliance and distensibility coefficients or the intima-media thickness of either the carotid and right common femoral arteries.

A recent study ${ }^{28}$ compared central systolic blood pressure (cSBP) and augmentation index (AIx) using two recently introduced devices, the Omron HEM-9000 and the Arteriograph, with those of the widely used SphygmoCor. Though heart rate is an important determinant of AIx, the heart rate adjusted AIx value was not statistically different across the three devices.

Some limitations of our study should be discussed. Firstly, this was not a double-blind randomized trial, and thus, the possibility of a significant bias cannot be excluded. However, all measurements were performed by a single clinical investigator who was blinded to the treatment allocation; therefore, there was no bias in the measurement and evaluation of laboratory data. Secondly, the study period was relatively short and the sample size was small; longer and larger studies are necessary to evaluate the long-term effects of smoking. In this study, a matched nonsmoker control group (individuals who did not smoke for at least more than 5 years or never smoked at all) was absent. Therefore, we cannot determine the time required for the vascular properties (CPB, baPWV, rAIx@75, etc) to return to normal. Thirdly, the measurement of $\mathrm{CO}$ levels to determine compliance with cessation is a weak measure because $\mathrm{CO}$ has a short half-life and therefore the estimation of cessation is weak.

\section{Conclusion}

Elevation in CBP, AI, and baPWV are strong risk factors for cardiovascular events. ${ }^{5}$ The present study revealed that smoking cessation can suppress CBP and AI. Thus, our results indicate the importance of smoking cessation as a primary approach for preventing cardiovascular events.

\section{Acknowledgment}

The authors wish to acknowledge Noriko Mune for technical support. No grants were used to fund this study.

\section{Disclosure}

The authors declare no conflicts of interest in this work.

\section{References}

1. Groppelli A, Giorgi DM, Omboni S, Parati G, Mancia G. Persistent blood pressure increase induced by heavy smoking. J Hypertens. 1992;10(5):495-499.

2. Minami J, Ishimitsu T, Matsuoka H. Effects of smoking cessation on blood pressure and heart rate variability in habitual smokers. Hypertension. 1999;33(1 Pt 2):586-590. 
3. Minami J, Ishimitsu T, Ohrui M, Matsuoka H. Association of smoking with aortic wave reflection and central systolic pressure and metabolic syndrome in normotensive Japanese men. Am J Hypertens. 2009;22(6):617-623.

4. Williams B, Lacy PS, Thom SM, et al. Differential impact of blood pressure-lowering drugs on central aortic pressure and clinical outcomes: principal results of Conduct Artery Function Evaluation (CAFÉ') study. Circulation. 2006;113(9):1213-1225.

5. Roman MJ, Devereux RB, Kizer JR, et al. Central pressure more strongly related to vascular disease and outcome than dose brachial pressure: the Strong Heart Study. Hypertension. 2007;50(1):197-203.

6. Pickering TG, Eguchi K, Kario K. Masked hypertension: a review. Hypertens Res. 2007;30(6):479-488.

7. Appel LJ, Brands MW, Daniels SR, Karanja N, Elmer PJ, Sacks FM. American Heart Association. Dietary approaches to prevent and treat hypertension: a scientific statement from the American Heart Association. Hypertension. 2006;47(2):296-308.

8. Van den Berkmortel FW, Wollersheim H, van Langen H, Smilde TJ, den Arend J, Thien T. Two years of smoking cessation does not reduce arterial wall thickness and stiffness. Neth J Med. 2004;62(7):235-241.

9. Kohara K. Central blood pressure, arterial stiffness and the heart in hypertensive patients. Hypertens Res. 2009;32(12):1056-1058.

10. Gonzales D, Rennard SI, Nides M, et al. Varenicline, an $\alpha 4 \beta 2$ nicotinic acetylcholine receptor partial agonist, vs sustained-release bupropion and placebo for smoking cessation. A randomized control trial. JAMA. 2006;296(1):47-55.

11. Rigotti NA, Pipe AL, Benowitz NL, Arteaga C, Carza D, Tonstad S. Efficacy and safety of varenicline for smoking cessation in patients with cardiovascular disease: a randomized trial. Circulation. 2010; 121(2):221-229.

12. Devereux RB, Palmieri V, Sharp N, et al. Effects of once-daily angiotensin-converting enzyme inhibition and calcium channel blockade-based antihypertensive treatment regimens on left ventricular hypertrophy and diastolic filling in hypertension: the prospective randomized enalapril study evaluating regression of ventricular enlargement (PRESERVE) trial. Circulation. 2001;104(11): 1248-1254.

13. Melenovsky V, Borlaug BA, Fetics B, Kesler K, Shively L, Kass DA. Estimation of central pressure augmentation using automated radial artery tonometry. J Hypertens. 2007;25(7):1403-1409.

14. Takazawa K, Kobayashi H, Shindo N, Tanaka N, Yamashina A. Relationship between radial and central artery pulse wave and evaluation of central aortic pressure using the radial artery pulse wave. Hypertens Res. 2007;30(3):219-228.
15. Richardson CJ, Maki-Petaja KM, McDonnell BJ, et al. Comparison of estimates of central systolic blood pressure and peripheral augmentation index obtained from the Omron HEM-9000AI and SphygmoCor systems. Artery Res. 2009;3:24-31.

16. Hickson SS, Butlin M, Mir FA, et al. The accuracy of central SBP determined from the second systolic peak of the peripheral pressure waveform. J Hypertens. 2009;27(9):1784-1788.

17. Wilkinson IB, Mohammad NH, Tyrrell S, et al. Heart rate dependency of pulse pressure amplification and arterial stiffness. Am J Hypertens. 2002;15(1 Pt 1):24-30.

18. Takami T. Evaluation of arterial stiffness in morning hypertension under high-dose valsartan compared to valsartan plus low-dose diuretic. Hypertens Res. 2009;32(12):1086-1090.

19. Soga J, Nakamura S, Nishioka K, et al. Relationship between augmentation index and flow-mediated vasodilation in brachial artery. Hypertens Res. 2008;31:1293-1297.

20. Reed DM, MacLean CJ, Hayashi T. Predictors of atherosclerosis in the Honolulu Heart Program. I. Biologic, dietary, and lifestyle characteristics. Am J Epidemiol. 1987;126(2):214-225.

21. Tsuchiya M, Thompson DF, Suzuki YJ, Cross CE, Packer L. Superoxide formed from cigarette smoke impairs polymorphonuclear leucocyte active oxygen generation activity. Arch Biochem Biophys. 1992;299(1):30-37.

22. Tsuchiya M, Asada A, Kasahara E, Sato EF, Shindo M, Inoue M. Smoking a single cigarette rapidly reduced combined concentrations of nitrate and nitrite and concentrations of antioxidants in plasma. Circulation. 2002;105(10):1155-1157.

23. Dzau VJ. Theodore Cooper Lecture: Tissue angiotensin and pathobiology of vascular disease: a unifying hypothesis. Hypertension. 2001;37(4):1047-1052.

24. Perkins KA, Epstein LH, Jennings JR, Stiller R. Cardiovascular effects of nicotine during stress. Psychopharmacology. 1986;90(3): 373-378.

25. Iida M, Iida H, Dohi S, Takenaka M, Fujiwara H. Mechanisms underlying cerebrovascular effects of cigarette smoking in rat in vivo. Stroke. 1998;29(8):1656-1665.

26. Kubozono T, Miyata M, Ueyama K, et al. Acute and chronic effects of smoking on arterial stiffness. Circ J. 2011;75(3):689-702.

27. Virdis A, Giannarelli C, Neves MF, Taddei S, Ghiadoni L. Cigarette smoking and hypertension. Curr Pharm Des. 2010;16(23):2518-2525.

28. Rezai MR, Goudot G, Winters C, Finn JD, Wu FC, Cruikshank JK. Calibration mode influences central blood pressure differences between SphygmoCor and two newer devices, the Arteriograph and Omron HEM-9000. Hypertens Res. 2011;34(9):1046-1051.
Vascular Health and Risk Management

\section{Publish your work in this journal}

Vascular Health and Risk Management is an international, peerreviewed journal of therapeutics and risk management, focusing on concise rapid reporting of clinical studies on the processes involved in the maintenance of vascular health; the monitoring, prevention and treatment of vascular disease and its sequelae; and the involvement of

\section{Dovepress}

metabolic disorders, particularly diabetes. This journal is indexed on PubMed Central and MedLine. The manuscript management system is completely online and includes a very quick and fair peer-review system, which is all easy to use. Visit http://www.dovepress.com/ testimonials.php to read real quotes from published authors. 